\title{
8 Realistisches, amimetisches und politisches Erzählen seit 1945
}

Um die aktuelle Konjunktur kontrafaktisch-politischer Literatur literarhistorisch angemessen bewerten zu können, erscheint es sinnvoll, sich zunächst die Geschichte des spannungsreichen Verhältnisses von (nicht-)realistischen Erzählverfahren und politischem Schreiben in der deutschsprachigen Literatur in aller Kürze vor Augen zu führen. Dass kontrafaktische, fantastische oder allgemein amimetische Erzählverfahren als Formen politischen Schreibens genutzt und als solche auch intensiv rezipiert werden, ist in der deutschsprachigen Literatur keineswegs eine Selbstverständlichkeit. Innerhalb der literarischen ebenso wie in der literaturwissenschaftlichen Diskussion nach 1945 wurden amimetische Erzählverfahren lange Zeit marginalisiert oder, wenn sie denn zur Diskussion gestellt wurden, eher mit Misstrauen beäugt. Noch im Oktober 2008 äußerte sich Dietmar Dath in einer FAZ-Rezension zu Christian Krachts Roman Ich werde hier sein im Sonnenschein und im Schatten in folgender Weise zum schwierigen Stand des nicht-realistischen Erzählens in der deutschsprachigen Literaturszene:

[Kracht] ist klar wie selten jemandem, dass er, wenn er Erzähltechniken der Phantastik in die deutsche Gegenwartsliteratur importiert, damit die Radarfallen der lizenzierten öffentlichen Literaturbetrachtung unterfliegt. Mehr noch als Humor nämlich diskreditiert Phantastik in Deutschland ihre Praktiker. Das ist ein literarisches Kuriosum, aber ein bitter wahres. Wenn ein paar Engländer in einem präzisen Zeitraum Geschichten über das erfinden, was kommen wird, oder über das, was nie war, entsteht eine Strömung namens Scientific Romance, mit weitreichenden Folgen; wenn ein paar Südamerikaner Ähnliches tun, spricht man von magischem Realismus; sind es Nordamerikaner, so entsteht sogar ein neues kommerzielles Genre, die Science-Fiction.

Wenn aber bei uns Ernst Kreuder eine Geheimgeschichte nach der anderen schreibt, wenn Arno Schmidt mehrere Zukunfts- und Unterweltepen dichtet, Wolf von Niebelschütz den größten Fantasyroman der Deutschen verfasst, Carl Amery und Wolfgang Jeschke das Kontrafaktische auf neue Wege führen oder Tobias O. Meißner der literarischen Fiktion die neuen Begrifflichkeiten der elektronischen Virtualität erschließt, dann sind das plötzlich alles Einzeltäter. Der befremdliche Tatbestand liegt letztlich daran, dass die populärsten Kunstgattungen allesamt phantastische waren und sind, wie jede ernsthafte Untersuchung Hollywoods, der Comics oder des Fernsehens zeigen müsste. Das Populäre als solches jedoch ist dem urteilenden Deutschlehrer ein Graus, ein Gelächter oder eine schmerzliche Scham. ${ }^{489}$

489 Dietmar Dath: Ein schöner Albtraum ist sich selbst genug. In: Frankfurter Allgemeine Zeitung, 15.10.2008.

Ә Open Access. (c) 2022 Michael Navratil, publiziert von De Gruyter. (cc) BY-NC-ND Dieses Werk ist lizenziert unter einer Creative Commons Namensnennung - Nicht-kommerziell - Keine Bearbeitung 4.0 International Lizenz. https://doi.org/10.1515/9783110763119-010 
Freilich handelt es sich bei Daths Polemik nicht zuletzt um eine Werbeschrift für den Kollegen Kracht, die mit ihrem Lob von Fantastik und Science-Fiction unter der Hand zugleich Daths eigene literarische Produktion aufwertet. Jenseits dieses pragmatisch-verkaufsstrategischen Aspekts sind Daths Ausführungen aber auch in literaturgeschichtlicher Hinsicht durchaus aufschlussreich. Dath konstatiert eine generelle Abwertung populärer Gattungen sowie fantastischer Erzählformen (kontrafaktisches Erzählen wird hier dem fantastischen Erzählen subsummiert) innerhalb der deutschen Literatur. Dabei lässt Dath die beiden Hochphasen der deutschsprachigen Fantastik in der deutschen Romantik - mit Autoren wie Ludwig Tieck, Joseph von Eichendorff und E. T. A. Hoffmann - sowie in der Frühen Moderne - Franz Kafka, Alfred Kubin, Gustav Meyrink und viele andere ${ }^{490}$ - außen vor und wählt stattdessen Beispiele der literaturgeschichtlichen Entwicklung im langen Nachgang der deutschsprachigen Nachkriegsliteratur. Während in anderen Kulturkreisen das amimetische Erzählen in hohen Würden stehe - Daths Beispiele sind die englische Scientific Romance, der südamerikanische Magische Realismus sowie die US-amerikanische Science-Fiction -, werden bedeutende Praktiker der utopischen, fantastischen oder kontrafaktischen Literatur in Deutschland kaum wahr- oder ernstgenommen. Einen Grund für diese Abwertung amimetischer Erzählverfahren sieht Dath dabei gerade in der Popularität derselben: Fantasy, Science-Fiction und Alternate History-Romane finden eine große Leserschaft - und werden, so die Implikation, aus just diesem Grund von den hochkulturellen Sachstandswahrern in den Zeitungsredaktionen und Universitäten geschmäht.

Tatsächlich hat die Abwertung der nicht-realistischen, insbesondere der fantastischen Kunst in Deutschland eine lange Tradition, die sich mindestens bis auf Goethes und Hegels Invektiven gegen die Schwarzen Romantik zurückverfolgen lässt. ${ }^{491}$ Marco Frenschkowski zufolge „mußte sich Phantastik [...] in den letzten

490 Vgl. Marianne Wünsch: Die Fantastische Literatur der Frühen Moderne (1890-1930). Auch Evelyne Jacquelin schreibt von „einem frühen Höhepunkt der Phantastik in der deutschsprachigen Romantik“ sowie einer „zweiten großen Epoche der deutschsprachigen Phantastik“ in der Zeit zwischen Décadence und Faschismus (Evelyne Jacquelin: Einleitung. In: Marie-Thérèse Moury / Evelyne Jacquelin (Hg.): Phantastik und Gesellschaftskritik im deutschen, niederländischen und nordischen Kulturraum / Fantastique et approches critiques de la société. Espaces germanique, néerlandophone et nordique. Heidelberg 2018, XI-XVIII, hier XVIII, XIV).

491 Vgl. Peter-André Alt: Ästhetik des Bösen. München 2010, S. 170-172; Wolfgang Preisendanz: Die geschichtliche Ambivalenz narrativer Phantastik in der Romantik. In: Athenäum. Jahrbuch für Romantik 2 (1992), S. 117-129, S. 118, Anm. 1. 
zwei Jahrhunderten mit drei Vorwürfen auseinandersetzen: Exzessivität, Trivialität und Eskapismus. “492 Für die Fragen nach dem Zusammenhang von amimetischem und politischem Schreiben ist speziell der letztgenannte Vorwurf von Interesse, die These also, fantastische Kunst verführe zu einer Flucht aus der Realität, inklusive all ihrer beunruhigenden Komplexität, moralischen Ambivalenzen und nicht zuletzt politischen Herausforderungen.

Definiert man politische Literatur über ihren Realitätsbezug, also über ihre Referenzen auf konkrete Ereignisse in der realen Welt - etwa in Geschichte, Gesellschaft oder Politik -, so scheint sich die Fantastik vorderhand tatsächlich wenig zur Verhandlung realweltlicher politischer Fragestellungen anzubieten; sehr viel naheliegender wäre eine Kopplung des politischen Schreibens an das realistische Erzählen. So bemerkt Evelyne Jacquelin:

Der Rückgriff auf imaginäre, kontraempirische Motive [...] kann den Verdacht des Eskapismus leicht aufkommen lassen [...]. Auch die Phantastik [...], die auf ihre Art mit den herkömmlichen Regeln der Mimesis spielt, ist über diesen Verdacht nicht erhaben. Auf den ersten Blick könnte man deshalb meinen, Phantastik sei zur Sozialkritik weniger geeignet als andere, in einem strengeren Maße realistische Darstellungsformen. Das Thema stand unter anderem in der zweiten Hälfte des 20. Jahrhunderts zur Debatte und verdient es, erneut diskutiert zu werden. ${ }^{493}$

Jacquelin eröffnet hier eine Opposition zwischen politischem Realismus auf der einen und fantastischem Eskapismus auf der anderen Seite. Dass der Sammelband, zu welchem Jacquelins Text die Einleitung bildet, die Wortkombination „Phantastik und Gesellschaftskritik“ im Titel trägt, weist freilich bereits darauf hin, dass die These von der notwendigen Depolitisierung der Fantastik nicht unangreifbar ist. ${ }^{494}$ Bei der Bindung des politischen Schreibens an den Realismus im Gegensatz zu einer apolitisch-amimetischen Kunst handelt es sich vor allem um ein Ordnungsnarrativ, das speziell für die deutschsprachige Diskussion des politischen Schreibens lange Zeit von großer Bedeutung war und teilweise bis in die Gegenwart hinein perpetuiert wird.

492 Marco Frenschkowski: Der Begriff des Phantastischen: Literaturgeschichtliche Beobachtungen. In: Ders. / Gerhard Lindenstruth / Malte S. Sembten (Hg.): Phantasmen. Robert N. Bloch zum Sechzigsten. Gießen 2010, S. 110-134, hier S. 127. Siehe zum Thema Eskapismus und Fantastik auch Hans-Christian Gebbe: Funktionen populärer Fantasy-Literatur in der christlichen Rezeption. Göttingen 2017, S. 15, Anm. 18, S. 94.

493 Jacquelin: Einleitung, XI.

494 Jacquelin weist in diesem Zusammenhang auf den schwierigen Stand der Fantastik in Deutschland, Finnland oder Schweden hin und betont, „dass gerade die sozialkritische und allgemeiner gesehen - auch ethische Tragweite der Texte immer wieder zitiert wird, um deren literarischen Wert zu behaupten." (Jacquelin: Einleitung, XVIII). 
Im Folgenden soll ein kurzer Abriss der Diskussion rund um das politische und apolitische respektive realistische und nicht-realistische Erzählen in der deutschsprachigen Literatur seit 1945 gegeben werden. Selbstredend erhebt diese literarhistorische Kurzdarstellung keinen Anspruch auf Vollständigkeit. ${ }^{495}$ Es wird lediglich der Versuch unternommen, die besondere Suggestivkraft einer Gegenüberstellung von politischem Realismus und fantastischem Eskapismus - und mithin den tendenziellen Ausschluss amimetischer Erzählverfahren aus dem Feld politischen Schreibens - innerhalb der deutschen Literaturgeschichte zu plausibilisieren. Mit Blick auf die Literatur etwa seit der Jahrtausendwende kann dann abschließend dargelegt werden, dass und weshalb die Opposition von politischem Realismus und fantastischem Eskapismus in der Gegenwart zusehends an Bedeutung verliert. ${ }^{496}$

Die deutsche Nachkriegsliteratur ist in ihren dominanten Strömungen durch eine starke Kopplung von politischem Schreiben und Realismus charakterisiert. So lässt sich bereits ein großer Teil der Texte aus dem Umfeld der Gruppe 47 dem Paradigma eines politischen Realismus zuschlagen. ${ }^{497}$ Sieht man einmal von der Lyrik ab, für welche die Unterscheidung von Realismus und Fantastik qua Gattung von untergeordneter Bedeutung ist ${ }^{498}$, so wird man konstatieren müssen, dass die zentralen Repräsentanten einer politisch orientierten oder zeitkritischen deutschsprachigen Nachkriegsliteratur vor allem als bedeutende Realisten bekannt sind: Man denke etwa an Siegfried Lenz, Heinrich Böll oder Uwe Johnson, später dann an Heiner Müller, Erika Runge oder Peter Weiss bis hin zu Rolf Hochhuth, Peter Handke und Botho Strauß. Die politisch ambitionierte Literatur

495 Es lassen sich hier allerdings leicht Themen und Fragestellungen für mögliche Anschlussforschungen ausmachen. Der Zusammenhang von politischem und fantastischem Erzählen in der Nachkriegsliteratur wäre ein überaus reizvolles Forschungsthema.

496 Die folgenden Ausführungen schließen an Überlegungen aus dem folgenden Aufsatz an: Navratil: Jenseits des politischen Realismus.

497 So bemerkt Anja Welle zur politischen Ausrichtung und den thematischen Präferenzen der Gruppe 47: „Von Anfang an war das Politische einbezogen, waren persönliche und künstlerische Gemeinsamkeiten nicht von politischen zu trennen [...]. Ein [...] Textkorpus, in den [sic] die Werke Hans Werner Richters, Alfred Anderschs und Günter Eichs ebenso einbezogen sind wie die Publikationen von Heinrich Böll, Günter Grass, Martin Walser, Hans Magnus Enzensberger und Uwe Johnson, zeigt sich thematisch dominiert von der Auseinandersetzung der Autoren mit der jüngsten Vergangenheit - viele fiktionale Texte von Gruppenmitgliedern handeln von Nationalsozialismus und Krieg.“ (Anja Welle: Die Gruppe 47 oder das ,Kritische Prinzip‘. In: Jürgen Wertheimer (Hg.): Von Poesie und Politik. Zur Geschichte einer dubiosen Beziehung. Tübingen 1994, S. 194-218, hier S. 195, S. 196f.).

498 Siehe zum ambivalenten Fiktionalitätsstatus von Lyrik Frank Zipfel: Lyrik und Fiktion. In: Dieter Lamping (Hg): Handbuch Lyrik. Theorie, Analyse, Geschichte. 2., erweiterte Aufl. Stuttgart 2016, S. 184-188. 
der DDR - etwa Texte von Christa Wolf, Jurek Becker oder Stefan Heym - lässt sich ebenso plausibel dem Paradigma eines ,politischen Realismus‘ zuschlagen wie ihr bundesrepublikanisches Pendant. Dass es sich auch beim sozialistischen Realismus um eine Form des politischen Realismus handelt, zeigt bereits der Begriff an. ${ }^{499}$

Zwar wurden auch in der deutschen Nachkriegsliteratur vereinzelt amimetische Erzählverfahren als Formen politischen Schreibens eingesetzt, etwa bei Arno Schmidt, Ingeborg Bachmann oder Leo Perutz. ${ }^{500}$ Die Tradition des Magischen Realismus, die in Deutschland bereits in den 1920er Jahren begonnen hatte, wurde nach 1945 unter anderem von Elisabeth Langgässer und Ilse Aichinger fortgeführt. ${ }^{501}$ Prominentestes Beispiel einer amimetischen Erzählliteratur mit politischem Anspruch aus den ersten Jahrzehnten der Bunderepublik ist zweifellos der Einsatz fantastischer und magisch-realistischer Elemente im Werk von Günter Grass, insbesondere im Roman Die Blechtrommel. Gegenüber dem dominanten Paradigma eines politischen Realismus erscheinen derartige Ausprägungen einer nicht-realistischen Kunst allerdings eher als Ausnahmen. Insgesamt ist die amimetische Literatur in der zweiten Hälfte des 20. Jahrhunderts vorwiegend ein Phänomen des englischsprachigen Kulturraums (abgesehen einmal vom Magi-

$499 \mathrm{Zu}$ nennen ist hier etwa die Literatur des ,Bitterfelder Weges‘, in dessen Rahmen eine stärkere Zusammenführung und gegenseitige Beeinflussung von Intellektuellen einerseits und vormals bildungs- und literaturferner Arbeiterschaft andererseits angestrebt wurde. Vgl. Jürgen Brokoff: Engagement und Schreiben zwischen Literatur und Politik. Zur Schreibreflexion in der Essayistik nach 1945 - mit einem Ausblick auf die Literatur der Arbeitswelt. In: Ders. / Ursula Geitner / Kerstin Stüssel (Hg.): Engagement. Konzepte von Gegenwart und Gegenwartsliteratur. Göttingen 2016, S. 227-248, hier S. $246 \mathrm{f}$.

500 Als Gegenstimmen zu den „schlichten Realismen“ der frühen Gruppe 47 benennt Heinz Ludwig Arnold neben Ingeborg Bachmann noch Ilse Aichinger und Paul Celan (Heinz Ludwig Arnold: Die Gruppe 47. Reinbek bei Hamburg 2004, S. 72f.). Das wechselvolle Rezeptionsschicksal des Werks von Leo Perutz ist im gegebenen Zusammenhang von besonderem Interesse: Der nach Palästina emigrierte Perutz setzte sein fantastisches Werk auch nach Ende des Zweiten Weltkriegs fort, wurde aber von Literaturkritik und lesender Öffentlichkeit kaum mehr wahrgenommen. Erst Ende der 1980er - also bezeichnenderweise in zeitlicher Parallele zur Renaissance des Erzählens in der deutschsprachigen Literatur - wurde Perutz' Werk wiederentdeckt und erfreut sich seither einer bescheidenen, aber kontinuierlichen Popularität.

501 Vgl. Torsten W. Leine: Magischer Realismus als Verfahren der späten Moderne. Paradoxien einer Poetik der Mitte. Berlin / Boston 2018, S. 287. In der zeitgenössischen poetologischen Diskussion wird der Magische Realismus - wie bereits der Begriff nahelegt - allerdings vor allem im Rahmen einer Poetik des politischen Realismus und weniger als dezidiert nicht-realistisches Erzählverfahren diskutiert. So bemerkt Leine: „Der Magische Realismus der Zwischenund Nachkriegszeit kann als besonders früher Entwurf betrachtet werden, eine politisch wie ästhetisch gemäßigte Position nach den Avantgarden zu besetzen, die eine wie auch immer gebrochene Rückkehr realistischer Zeichengebungsverfahren ermöglicht.“ (ebd., S. 294f.). 
schen Realismus moderner südamerikanischer Autoren und der metaphysischen Fantastik von Jorge Luis Borges). ${ }^{502}$

Die tendenzielle Verweigerung gegenüber der amimetischen Kunst, die vor dem Zweiten Weltkrieg ja noch durchaus hoch im Kurs gestanden hatte, innerhalb der Gruppe 47 und darüber hinaus dürfte sich vor allem aus dem „,besondere[n] Profil“ der deutschen Nachkriegsliteratur erklären lassen, nämlich ihrer Selbstverpflichtung auf „moralische Schuldreflexion und den Aufbau einer besseren Gesellschaft““. ${ }^{503}$ Dieses politische Sendungsbewusstsein verpflichtete die Nachkriegsliteratur auf eine enge Bindung an die eigene Gegenwart und Vergangenheit; der Entfaltung freischwebender schriftstellerischer Fantasie dürfte es hingegen wenig zuträglich gewesen sein. So konstatiert Heinz Schlaffer in seiner Kurzen Geschichte der deutschen Literatur:

Politisches Engagement gehört seit 1945 zum Metier des deutschen Schriftstellers - im Westen wie im Osten, denn Stil und Haltung der Autoren in der Bundesrepublik und in der DDR unterschieden sich sehr viel weniger als die politischen Systeme, in denen sie lebten [...]. Beim einen wie beim anderen Typus der deutschen Literatur nach 1945, dem offenen wie dem verdeckten Moralismus, blockiert die Angst vor der Verführung durch die Phantasie (denn wer weiß, wohin sie wieder führen könnte?) das eigentliche ästhetische Vermögen. Undeutbare Geschichten, befremdliche Bilder, riskante Gedanken, unvorhersehbare Experimente in einer entfesselten Sprache - jene Freuden also, wie sie die süd- und nordamerikanische Literatur in der zweiten Hälfte des 20. Jahrhunderts bereitet, muß der Leser der deutschen Gegenwartsliteratur vermissen. ${ }^{504}$

Auch die Tendenzen der philosophischen und ästhetischen Diskussion in der Nachkriegszeit waren einem Wiedererstarken amimetischer Erzähltraditionen wenig förderlich. So betonte Theodor W. Adorno zwar emphatisch die gesellschaftliche Relevanz einer nicht-realistischen Kunst; das wünschenswerte Gegenmodell $\mathrm{zu}$ einem naiven Realismus erblickte Adorno allerdings nicht in der Fantastik, sondern vor allem in der modernistischen, formal verrätselten Kunst der historischen Avantgarden. ${ }^{505}$ Tatsächlich stellten Adorno und Horkheimer

502 Vgl. Rottensteiner: Eine kurze Geschichte der Phantastischen Literatur, S. 107.

503 Willi Huntemann: „Unengagiertes Engagement“ - zum Strukturwandel des literarischen Engagements nach der Wende. In: Willi Huntemann / Małgorzata Klentak-Zabłocka / Fabian Lampart / Thomas Schmidt (Hg.): Engagierte Literatur in Wendezeiten. Würzburg 2003, S. 33-48, hier S. 38.

504 Heinz Schlaffer: Die kurze Geschichte der deutschen Literatur. München / Wien 2002, S. 149 f. 505 Eher im Vorübergehen geht Adorno in der Ästhetischen Theorie auf die Fantastik ein. Adorno verwahrt sich dabei gegen die Charakterisierung der Kunst der Moderne als fantastisch: „Kafkas Kraft schon ist die eines negativen Realitätsgefühls; was an ihm dem Unverstand phantastisch dünkt ist ,Comme c'est‘. Durch غ́лoxท́ von der empirischen Welt hört die neue Kunst auf, phantastisch zu sein.“ (Theodor W. Adorno: Ästhetische Theorie. Frankfurt a. M. 1970, S. 36) Siehe zu Adornos Realismus-Verständnis überblicksweise Karol Sauerland: 
die Beschäftigung mit populären Kunstformen, wie sie gerade im Genrebereich des amimetischen Erzählens häufig anzutreffen sind, unter den Generalverdacht der „Kulturindustrie“. ${ }^{506}$

Als wirkmächtigste philosophische Einlassung zum Zusammenhang von Literatur und Politik wurde Jean-Paul Sartres Essay Qu'est-ce que la littérature von 1948 in Deutschland seit den 1960er Jahren verstärkt rezipiert. ${ }^{507}$ In diesem Text entfaltete Sartre seine Ideen zur ,littérature engagée ' vorwiegend anhand von Beispielen des realistischen Erzählens. Sartres bedenkenswerte Überlegungen zur Fantastik hingegen wurden - und werden selbst noch heute - in Deutschland kaum wahrgenommen. ${ }^{508}$

Nach dem Ende des Kalten Krieges avancierte die Verbindung von politischem Schreiben und einer bestimmten Form von uninspiriertem Realismus zu einem zentralen Streitpunkt im sogenannten deutsch-deutschen Literaturstreit: Frank Schirrmacher formulierte 1990 seinen „Abschied von der Literatur der Bundesrepublik“, die er in dem moralisch-ästhetischen Doppelanspruch der Nachkriegsjahre unproduktiv festgefahren sah. ${ }^{509}$ In großer argumentativer Nähe zu Schirrmacher wandte sich Ulrich Greiner 1990 gegen die „Gesinnungsästhetik“ der deutschen Nachkriegsliteratur, die er als eine „Variante des deutschen Sonderweges“ diffamierte. ${ }^{510}$ Parallel zum deutsch-deutschen Literaturstreit wurden in den Feuil-

Einführung in die Ästhetik Adornos. Berlin / New York 1979 (Reprint 2019), darin das Kapitel XXI. Kunst und Realität, S. 135-151.

506 Siehe zur „Kulturindustrie“ Theodor W. Adorno / Max Horkheimer: Dialektik der Aufklärung. Frankfurt a. M. ${ }^{20} 2011$, S. $128-176$.

507 Die Literatur der jungen Bundesrepublik wird in literaturgeschichtlichen Studien häufig mit dem Konzept des ,Engagements' respektive der ,engagierten Literatur' assoziiert. Sofern damit ein politischer Anspruch der Literatur respektive der Schriftsteller gemeint ist, mag man diese Charakterisierung akzeptieren; der Begriff, Engagement' selbst jedoch spielt innerhalb der poetologischen Selbstverortungen der Autoren zunächst kaum eine Rolle. Für die frühen Jahre der Gruppe 47 spricht Helmut Peitsch gar von einer „Verweigerung der Rezeption von Sartres Konzept“ (Helmut Peitsch: Die Gruppe 47 und das Konzept des Engagements, S. 25).

508 Vgl. Jean-Paul Sartre: ,Aminadab“ oder Das Phantastische als Ausdrucksweise betrachtet. In: Ders.: Situationen. Essays. Hamburg 1965, S. 143-156.

509 „Die westdeutsche Literatur wurde dreiundvierzig Jahre alt. Sie war das Werk einer Generation. [...] Genötigt von einer sich schuldig fühlenden Gesellschaft, zu bessern, zu belehren und zu erziehen, aufgefordert, ein demokratisches Bewußtsein zu beweisen, fanden sich die Schriftsteller in einer schier ausweglosen Situation." (Frank Schirrmacher: Abschied von der Literatur der Bundesrepublik. In: Frankfurter Allgemeine Zeitung, 02.10.1990. Wiederabgedruckt in ders.: Ungeheuerliche Neuigkeiten. Texte aus den Jahren 1990 bis 2014. München 2014, S. 271-281, hier S. 279).

510 „Die Gesinnungsästhetik hat eine zutiefst deutsche Tradition. Sie wurzelt in der Verbindung von Idealismus und Oberlehrertum. Sie ist eine Variante des deutschen Sonderwegs. Sie läßt der Kunst nicht ihr Eigenes, sondern verpflichtet sie (wahlweise) auf die bürgerliche 
letons erhitzte Realismusdebatten geführt. ${ }^{511} 1991$ erklärte Maxim Biller den Realismus als „lebensnotwendig“ für die Literatur und mahnte eine stärkere Verbindung von journalistischem und literarischem Schreiben an, um „die gute, ernste Literatur ins Bewußtsein der Leser zurückzuführen“. ${ }^{512}$ Roger Willemsen hingegen polemisierte 1992 gegen den „,Always Ultra'-Realismus, der kein Jugoslawien, kein Somalia, keinen Bundestag und keine Straßenschlachten kennt“, und forderte stattdessen einen „Realismus der Evidenz [...], der im Satz Fakten schafft, so wie der Selbstmord der Madame Bovary keine Widerspiegelung einer Katastrophe ist, sondern diese selbst. “513 Als eine der prominentesten programmatischen Verbindungen von Realismus und politischem Schreiben kann ein im Jahre 2005 in der Zeit veröffentlichter Artikel von Matthias Politycki, Martin R. Dean, Thomas Hettche und Michael Schindhelm mit dem Titel Was soll der Roman? gelten. Die Autoren fordern darin eine Distanzierung der Gegenwartsliteratur sowohl von den „großmäuligen Alten, [den] Deutungshoheiten mit und ohne Pfeife“ (die Grass-Schmähung ist hier kaum kaschiert) als auch von den „Dienstleister[n] gestriegelter Populärliteratur“. Stattdessen plädieren sie für einen ,Relevanten Realismus‘, der Gegenwartsanbindung mit politischer Relevanz verbindet. ${ }^{514}$ Der Regisseur Bernd Stegemann schließlich gab 2015 in seinem Buch Lob des Realismus der Hoffnung Ausdruck, dass „gerade die Kunst des Theaters durch den Realismus wieder zu einem Selbstbewusstsein

\footnotetext{
Moral, auf den Klassenstandpunkt, auf humanitäre Ziele oder neuerdings auf die ökologische Apokalypse. Die Gesinnungsästhetik [...] ist das gemeinsame Dritte der glücklicherweise zu Ende gegangenen Literaturen von BRD und DDR.“ (Ulrich Greiner: Die deutsche Gesinnungsästhetik. In: Die Zeit, 02.11.1990. Nachgedruckt in Thomas Anz (Hg.): „Es geht nicht um Christa Wolf“. Der Literaturstreit im vereinten Deutschland. München 1991, S. 208-216, hier S. 213).

511 Vgl. Richard Kämmerlings: Das kurze Glück der Gegenwart. Deutschsprachige Literatur seit '89. Stuttgart 2011, S. 27. Siehe zu den feuilletonistischen Realismus-Debatten seit 1990 auch Leonhard Herrmann: Andere Welten - fragliche Welten. Fantastisches Erzählen in der Gegenwartsliteratur. In: Silke Horstkotte / Leonhard Herrmann (Hg.): Poetiken der Gegenwart. Deutschsprachige Romane nach 2000. Berlin / Boston 2013, S. 47-65, hier S. $48 \mathrm{f}$.

512 Maxim Biller: Soviel Sinnlichkeit wie der Stadtplan von Kiel. In: Die Weltwoche, 25.07.1991. Nachgedruckt in Andrea Köhler / Rainer Moritz (Hg.): Maulhelden und Königskinder. Zur Debatte über die deutschsprachige Gegenwartsliteratur. Leipzig 1998, S. 62-71, hier S. 69 f.

513 Roger Willemsen: Fahrtwind beim Umblättern. In: Der Spiegel, 21.12.1992. Nachgedruckt in Andrea Köhler / Rainer Moritz (Hg.): Maulhelden und Königskinder. Zur Debatte über die deutschsprachige Gegenwartsliteratur. Leipzig 1998, S. 79-85, hier S. 84 f.

514 Vgl. Matthias Politycki u. a.: Was soll der Roman? Der durchaus polemische Artikel von Politycki und Kollegen provozierte journalistische Stellungnahmen von Andreas Maier, Uwe Tellkamp, Hans-Ulrich Treichel und Juli Zeh.
} 
zurückfindet“" ${ }^{515}$, und erinnerte in diesem Zusammenhang an die traditionsreiche Verbindung von künstlerischem Realismus und politischem Anspruch. ${ }^{516}$

Eine detaillierte Analyse der genannten Positionen würde freilich zeigen, dass mit dem Begriff ,Realismus' hier im Detail zum Teil sehr Verschiedenes bezeichnet ist. Gemeinsam ist den vorgestellten Positionen jedoch bei aller Verschiedenheit die Kopplung des Politischen in der Literatur an eine mimetische Form des Realitäts- und Gegenwartsbezugs. Auf die ein oder andere Weise hat man es hier also jeweils mit Konzeptionen eines politischen Realismus zu tun. Demgegenüber wird die Möglichkeit einer politisch relevanten, dabei aber amimetisch, fantastisch oder kontrafaktisch erzählenden Literatur kaum - oder eher gar nicht - in Erwägung gezogen.

Die Opposition von politischem Realismus auf der einen und fantastischem Eskapismus auf der anderen Seite scheint sich ex negativo auch anhand jener literarischen Entwicklungen zum Ende des zwanzigsten Jahrhunderts zu bestätigen, die sich ihrerseits dezidiert vom politischen Realismus der Nachkriegsliteratur zu distanzieren suchten. Seit den 1980er Jahren ließen sich in der Literatur jüngerer deutschsprachiger Autoren deutliche Abgrenzungsbewegungen gegenüber den Erzählverfahren und politischen Tendenzen der etablierten deutschsprachigen Nachkriegsliteratur beobachten. Die Autoren der ,Wiederkehr“ oder ,Renaissance des Erzählens ${ }^{517}$ seit den 1980er Jahren - etwa Patrick Süskind, Robert Schneider oder Christoph Ransmayr -, die Autorinnen des sogenannten ,Fräuleinwunders', die Autoren der neuen deutschen Popliteratur bis hin zu zeitgenössischen Bestsellerautoren wie Helmut Krausser oder Daniel Kehlmann wandten sich allesamt sowohl vom politischen Moralismus als auch von der häufig als provinziell-nachkriegsdeutsch und ästhetisch mutlos empfundenen Formkonzeption einer realistischpolitischen Kunst ab. Stattdessen suchten diese Autoren den Schulterschluss mit den ästhetischen Entwicklungen einer internationalen, vor allem englischspra-

515 Bernd Stegemann: Lob des Realismus. Berlin 2015, S. 203.

516 In der Einleitung seines Buches schreibt Stegemann: „Alle künstlerischen Realismen waren sich in zwei Punkten einig: Es gibt eine Realität, und wir können versuchen, sie zu verstehen. Und es gibt eine künstlerische Erfahrung, die den Menschen ein gemeinsames Erleben ermöglicht, das sie momentweise davon befreit, ihr eigenes Leben als unverständliche Folge von Zufällen zu erleiden. Eine realistische Darstellung hilft, die Welt begreifen und sich ihre Veränderbarkeit vorstellen zu können.“ (Stegemann: Lob des Realismus, S. 8).

517 Siehe allgemein zur Renaissance respektive Wiederkehr des Erzählens Nikolaus Förster: Die Wiederkehr des Erzählens. Deutschsprachige Prosa der 80er und 90er Jahre. Darmstadt 1999; Gerd Herholz (Hg.): Experiment Wirklichkeit. Renaissance des Erzählens? Poetikvorlesungen und Vorträge zum Erzählen in den 90er Jahren. Essen 1998; Helmut Gollner: Die Wahrheit lügen. Die Renaissance des Erzählens in der jungen österreichischen Literatur. Innsbruck 2005. 
chigen Moderne und Postmoderne, mit ihrem sehr viel stärkeren Nachdruck auf Lesbarkeit $^{518}$, Unterhaltsamkeit, aber auch ihren größeren Lizenzen zur freien literarischen Erfindung: etwa im historischen Roman, in Fantasy und Science-Fiction oder im Magischen Realismus südamerikanischer Prägung - in Genres also, bei denen man in Deutschland oftmals den Hautgout des Trivialen wahrzunehmen meinte. Der zunehmende Erfolg derartiger populärer Genres und Erzählverfahren in den 1990er Jahren lief dabei einer generellen Depolitisierung der jüngeren Gegenwartsliteratur parallel: Bedeutende politische ,Einmischungen' wurden in den 1990er Jahren vor allem von Autoren der älteren Generation vollbracht: Man denke an Botho Strauß’ Essay Anschwellender Bocksgesang (1993), an die Kontroverse um Peter Handkes Reisebericht Eine winterliche Reise zu den Flüssen Donau, Save, Morawa und Drina oder Gerechtigkeit für Serbien (1996) oder an Martin Walsers Rede zur Verleihung des Friedenspreises des deutschen Buchhandels (1998). Weite Teile der jungen deutschsprachigen Literatur hingegen distanzierten sich von der Idee einer engagierten Literatur und der Rolle des öffentlichen Intellektuellen: So wandte sich die neue deutsche Pop-Literatur, anstatt sich weiterhin primär mit den Schrecken der Vergangenheit zu befassen, auf emphatische Weise der Gegenwart zu. ${ }^{519}$ Auch die ,Renaissance des Erzählens` lässt sich hinsichtlich ihrer dominanten Erzählverfahren und ihrem Verzicht auf politische Relevanzprätentionen als Abgrenzungsbewegung gegenüber der deutschsprachigen Nachkriegsliteratur verstehen. ${ }^{520}$ Insgesamt zeichnen sich die 1990er Jahre durch eine „Delegitimierung von Engagement in Literatur und Literaturwissenschaft“"521 aus. Der Verleger Klaus Wagenbach etwa formulierte 1998 kernig, dass sich „[m]it der Wiedervereinigung der beiden immer noch ziemlich getrennten deutschen Staaten [...] ein gemeindeutscher Ekel gegenüber der ,engagierten Literatur` breitgemacht [habe]“. 522

518 Matthias Politycki führte den Begriff der „Lesbarkeit“ 1995 in die literaturkritische Debatte ein, als er einen „Durchbruch der „Neuen Deutschen Lesbarkeit““ für die deutschsprachige Gegenwartsliteratur konstatierte (Matthias Politycki: Neue Äusserlichkeit. In: Ders.: Die Farbe der Vokale. Von der Literatur, den 78ern und dem Gequake satter Frösche. München 1998, S. 5-10, hier S. 5). Siehe hierzu auch Benjamin Schaper: Poetik und Politik der Lesbarkeit in der deutschen Literatur. Heidelberg 2017, S. 93-116.

519 Vgl. Moritz Baßler: Der deutsche Pop-Roman. Die neuen Archivisten. München 2002, S. 14 f. 520 Vgl. Förster: Die Wiederkehr des Erzählens, S. 5 f.

521 Helmut Peitsch: ,Vereinigungsfolgen'. Strategien zur Delegitimierung von Engagement in Literatur und Literaturwissenschaft der neunziger Jahre. In: Weimarer Beiträge 3/47 (2001), S. 325-351. 522 Klaus Wagenbach: Das Ende der engagierten Literatur? In: Neue deutsche Literatur 46/4 (1998), S. 193-201, hier S. 193. Siehe hierzu auch Peitsch: Zur Rolle des Konzepts ,Engagement in der Literatur der 90er Jahre. 
Der postpolitischen Abspannung in Gesellschaft und Literatur der 1990er Jahre - man denke an Francis Fukuyamas notorische Proklamation vom ,Ende der Geschichte ${ }^{623}$ - wurde durch die Anschläge auf das World Trade Center am 11. September 2001 ein symbolisch eindrückliches sowie weltpolitisch folgenreiches Ende bereitet. Nach der Jahrtausendwende fand auch die deutschsprachige Literatur verstärkt zum Politischen zurück, sodass die ,Renaissance des Erzählens“ nun wiederum von einer „Renaissance des Engagements“ abgelöst wurde. ${ }^{524}$ In Feuilleton und Literaturwissenschaft wurde zu Beginn des neuen Jahrhunderts zwar noch verschiedentlich - und bald geradezu reflexhaft - ein Desinteresse der Gegenwartsliteratur an politischen Fragestellungen konstatiert; bei dieser Klage dürfte es sich jedoch vor allem um einen nachgereichten Kommentar zur Literatur der 1990er Jahre gehandelt haben, ein Kommentar, der neuere Entwicklungen in der Gegenwartsliteratur entweder unberücksichtigt ließ oder strategisch ausschloss. ${ }^{525}$ Frank Schirrmacher etwa bemerkte in einem FAZ-Artikel vom März 2011: „In den siebziger und achtziger Jahren war die Literatur, waren die Schriftsteller in hohem Maße politisch engagiert. Dann wurde das Engagement wohlfeil und starb ab. Nun bietet unsere Gegenwart Gründe zuhauf, um das Politische poetisch wiederzugewinnen.“ Allerdings mutet Schirrmachers Befund von der „,völ-

523 Die Distanzierung von politischen Inhalten in der Literatur der 1990er Jahre bildet nicht zuletzt einen Reflex auf eine generell depolitisierte Stimmung der Zeit: Das Ende des Kalten Krieges wirkte für weite Teile der westlichen Gesellschaften als Befreiungsschlag im Bereich des Politischen - und zugleich als Befreiung vom Politischen. Francis Fukuyama brachte 1989 den Begriff vom „Ende der Geschichte“ in die Diskussion ein. Vgl. Francis Fukuyama: The End of History? In: The National Interest 16 (1989), S. 3-18. Toni Blair und Gerhard Schröder bezeichneten 1999 in einem gemeinsamen Paper „[f]lexible Märkte“ als „ein modernes sozialdemokratisches Ziel“ und versuchten damit die traditionelle Spannung zwischen Sozialismus und Kapitalismus in einer - letztlich neoliberalen - Sozialdemokratie des ,dritten Weges` aufzulösen. Vgl. Toni Blair / Gerhard Schröder: Der Weg nach vorne für Europas Sozialdemokraten. Quelle: http://www.glasnost.de/pol/schroederblair.html (Zugriff: 27.07.2021). Die großen Wirtschaftskrisen, die den globalisierten Markt nachhaltig erschüttern sollten, setzten erst nach der Jahrtausendwende ein.

524 Brokoff / Geitner / Stüssel: Einleitung, S. 9.

525 Vgl. Tanja Dückers: Die Mär von den jungen unpolitischen Autoren. In: EDIT 29 (2002). Quelle: http://www.tanjadueckers.de/die-mar-von-den-jungen-unpolitischen-autoren/ (Zugriff: 27.07.2021). Auch Thomas Wagner bezeichnet „das Gerede vom Verstummen der engagierten Literatur“ als ein „Märchen“ und betont demgegenüber: „Zwanzig Jahre nach dem Mauerfall ist die Literatur so breit und vielgestaltig engagiert wie schon lange nicht mehr. Jenseits von Pop-Literatur, Fräuleinwunder und dem sogenannten Neuen Feminismus melden sich Autorinnen und Autoren deutlich vernehmbar zu Wort, greifen Schriftsteller als kritische Intellektuelle kraftvoll und beherzt in die gesellschaftlichen Auseinandersetzungen ein.“ (Wagner: Die Einmischer, S. 5f.). 
lige[n] Entpolitisierung von Literatur und literarischem Leben“ mit Blick auf die Werke etwa von Juli Zeh, Thomas Meinecke, Kathrin Röggla, Elfriede Jelinek, Ilija Trojanow, Robert Menasse oder Christian Kracht in der Rückschau einigermaßen kurios an. ${ }^{526}$

Dass die deutschsprachige Gegenwartsliteratur sich wieder verstärkt mit politischen Fragestellungen auseinandersetzt, kann seit Ende der 2010er Jahre auch innerhalb der Literaturwissenschaft als Konsens gelten. Christine Lubkoll, Manuel Illi und Anna Hampel etwa beobachten eine „Hochkonjunktur politischer Inhalte (Jenny Erpenbeck, Abbas Khider, Robert Menasse, Sasha Marianna Salzmann, Eugen Ruge), poetologischer Reflexionen (Ulrike Draesner, Kathrin Röggla, Ilija Trojanow) und medial inszeniertem politischem Engagement (Juli Zeh, Navid Kermani) in der gegenwärtigen Literaturlandschaft. “527 Freilich beruht die Rede vom Erstarken einer ,politischen Gegenwartsliteratur' nicht auf der quantitativen Auszählung objektiver Kriterien in einem möglichst großen Textkorpus. Stattdessen handelt es sich hier um Beobachtungen von Diskurskonjunkturen sowie um Tendenzaussagen, die immer nur für einen bestimmten Teil der literarischen Produktion zutreffen. ${ }^{528}$ In jedem Falle bleibt festzuhalten: Ein fehlendes Interesse an politischen Themen und Fragestellungen wird man der jüngeren deutschsprachigen Literatur grosso modo kaum mehr plausibel vorwerfen können.

Blickt man auf das Verhältnis von realistischem, amimetischem und politischem Erzählen, so lässt sich zunächst festhalten, dass auch in der Gegenwartsliteratur Realismus und politisches Schreiben weiterhin in enger Verbindung zueinander stehen: Die hohe mediale Beachtung und das politische Diskussionspotenzial etwa von Werken wie David Wagners Leben, Benjamin von Stuckrad-Barres Panikherz, Saša Stanišićs Herkunft oder der Werke von Édouard Louis lassen sich wesentlich auf ihre Einbeziehung konkreter gesellschaftlicher Realität und persönlicher Erfahrungen der Autoren zurückführen. ${ }^{529}$ Auch geht die rezente Konjunktur politischen Schreibens mit einer verstärkten Diskussion und Aufwertung des Realismus respektive mit

526 Schirrmacher: Literatur und Politik. Eine Stimme fehlt.

527 Lubkoll / Illi / Hampel: Einleitung, S. 1.

528 Zurecht betont Kristin Eichhorn, dass es „[n]ach wie vor [...] Autoren [...] gibt, die den allerorts konstatierten Trend zu mehr Ernsthaftigkeit nicht mitmachen und deren Hauptinteresse ungebrochen auf dem metafiktionalen und intertextuellen Spiel liegt." (Kristin Eichhorn: Einleitung: Reaktionen auf den Ernsthaftigkeitsdiskurs in der aktuellen Literaturproduktion. In: Dies. (Hg.): Neuer Erst in der Literatur? Schreibpraktiken in deutschsprachigen Romanen der Gegenwart. Frankfurt a. M. 2014, S. 9-14, hier S. 10).

529 Siehe zum Boom autofiktionalen Erzählens in der jüngeren Gegenwartsliteratur Johannes Franzen: Hemmung vor der Wirklichkeit. In: Zeit online, 15.10.2019, Quelle: https://www.zeit. de/kultur/literatur/2019-10/literaturkritik-fiktion-fakten-schreiben-qualitaet/komplettansicht (Zugriff: 27.07.2021). 
der Ausrufung eines ,neuen Realismus“ in Literaturwissenschaft und Philosophie einher oder läuft dieser zumindest parallel. ${ }^{530}$

Gleichwohl hat die Konjunktur des Realismus zu Beginn des 21. Jahrhunderts nicht zu einem Abflachen der „postmoderne[n] Phantastik-Konjunktur“ geführt. ${ }^{531}$ Ganz im Gegenteil lässt sich in der jüngeren deutschsprachigen Gegenwartsliteratur geradezu ein Boom unterschiedlichster Ausprägungen amimetischen Erzählens beobachten: von der historischen Kontrafaktik (Christian Kracht, Christoph Ransmayr, Andreas Eschbach, Thomas Brussig) und Fantastik (Daniel Kehlmann, Sibylle Lewitscharoff, Cornelia Funke) über Utopie und Dystopie (Juli Zeh, Leif Randt, Karen Duve) bis hin zur Science-Fiction (Dietmar Dath, Reinhard Jirgl, Georg Klein) und literarischen Apokalyptik (Thomas Glavinic, Alban Nicolai Herbst, Thomas Lehr). ${ }^{532}$ Die von Dietmar Dath 2008 formulierte Klage, dass „Phantastik in Deutschland ihre Praktiker [diskreditiert]“, scheint mittlerweile selbst historisch geworden zu sein. Ende der 2010er Jahre stehen amimetische Erzählverfahren in der deutschsprachigen Gegenwartsliteratur so hoch im Kurs wie wohl zuletzt vor etwa einhundert Jahren in der Frühen Moderne und davor das letzte Mal wiederum einhundert Jahren früher in der deutschen Romantik.

Unverkennbar ist der aktuelle Fantastik-Boom im Bereich der Populärkultur. Moritz Baßler bemerkt hierzu:

Harry Potter, die Renaissance vom Lord of the Rings und den Chroniken von Narnia, Star Wars, Artemis Fowl, zahlreiche Kinder- und Jugendbücher, dann die japanischen Varianten von den Pokémons über den magischen Realismus der Mangas und Animes bis hin zur global erfolgreichen Literatur Haruki Murakamis, dazu die ganze Welt der Rollenspiele, des Gothic, der Cyberromane usw. - es gehört nicht viel zu der Prognose, dass unsere Zeit, was ihre Epik betrifft, als Zeitalter der Fantasy in die Geschichte eingehen wird. ${ }^{533}$

530 In den letzten Jahren ist der Realismus wieder verstärkt Thema der Diskussion geworden. Siehe etwa Baßler: Populärer Realismus; Markus Gabriel (Hg.): Der Neue Realismus. Berlin 2014; Søren R. Fauth / Rolf Parr (Hg.): Neue Realismen in der Gegenwartsliteratur. Paderborn 2016. Zu den Schwierigkeiten eines Bestimmung des ,neuen Realismus‘ in der Gegenwartsliteratur siehe Michael Navratil: Rezension zu: Søren R. Fauth / Rolf Parr (Hg.): Neue Realismen in der Gegenwartsliteratur. Paderborn 2016. In: Komparatistik. Jahrbuch der Deutschen Gesellschaft für Allgemeine und Vergleichende Literaturwissenschaft 2017. Bielefeld 2018, S. 288-294. 531 Brittnacher / May: Phantastik-Theorien, S. 192.

532 Siehe einführend zum amimetischen Erzählen in der Gegenwartsliteratur Herrmann / Horstkotte: Gegenwartsliteratur. Eine Einführung, S. 143-160.

533 Baßler: Moderne und Postmoderne, S. 448. 
Baßlers Ausführungen zur Fantasy scheinen zunächst Daths These zu bestätigen, „dass die populärsten Kunstgattungen allesamt phantastische waren und sind“. Während Dath jedoch für eine Kanonisierung der fantastischen Literatur als relevantem Teil der Kunstproduktion plädiert, steht Baßler dem aktuellen Boom der Fantasy kritisch gegenüber: Für Baßler bildet die Fantasy den paradigmatischen Fall einer epischen Kunst der Gegenwart, die mühelos rezipierbar ist, da sie auf modernistische Verfahren der formalen Verrätselung verzichtet und sich stattdessen bestens bekannter Scripts und Frames bedient, und zwar auch und gerade da, wo die Inhalte dieser Frames - Drachen, Zauberer, Hobbits etc. - fiktiv sind. ${ }^{534}$ (Eher contra-intuitiv bezeichnet Baßler solche mühelos rezipierbaren Erzählverfahren als „Realismus“. ${ }^{535}$ ) Den Fluchtpunkt zeitgenössischer Fantastik sieht Baßler dabei in einem „Realismus ohne Wirklichkeit“536, will sagen: in der Erzeugung stoff- und handlungsreicher Erzählwelten, die bei der Lektüre mühelose rezipierund imaginierbar sind, dabei jedoch kaum noch eine Beziehung zur empirischen Realität der Leser unterhalten: „Fantasywelten und -geschichten sind keine Allegorien. [...] Fantasywelten sind Simulationen, ja, aber nicht von Aspekten unserer Welt. “537 Baßler zufolge hat man es in der Fantastik mit „neuen, attraktiven, eigenständigen, besonders bewohnbaren Welt[en]“ $\mathrm{zu}$ tun, Welten also, die ontologisch sowie moralisch sehr viel klarer geordnet sind als jene Welt, welche die realen Leser zu bewohnen gezwungen sind. Erkennbar hallt hier ein weiteres Mal der altbekannte Eskapismusvorwurf gegenüber der fantastischen Kunst wider.

Nun wird man Baßlers These von der Realitätsentrücktheit fantastischer Welten mit Blick auf die fantastische Genre- und Unterhaltungsliteratur wohl weitge-

534 „Nur über die allerstabilsten Frames und Codes werden die fantastischen Dinge als solche lesbar und damit zu den Regeln einer roman- oder genrespezifischen Wirklichkeit.“ (Moritz Baßler: Realismus - Serialität - Fantastik, S. 35) Siehe zu dem diesen Ausführungen zugrunde liegenden, verfahrensanalytisch-strukturalistischen Modell Baßler: Deutsche Erzählprosa 1850-1950, S. 11-30.

535 „Realistisches Erzählen als metonymisches Verfahren setzt dem Verstehen keinen Widerstand entgegen.“ (Moritz Baßler: Die Unendlichkeit des realistischen Erzählens. Eine kurze Geschichte moderner Textverfahren und die narrativen Optionen der Gegenwart. In: Carsten Rohde / Hansgeorg Schmidt-Bergmann (Hg.): Die Unendlichkeit des Erzählens. Der Roman in der deutschsprachigen Gegenwartsliteratur seit 1989. Bielefeld 2013, S. 27-45, hier S. 27) An anderer Stelle schreibt Baßler: „,[M]it Realismus ist zunächst ein Verfahren bezeichnet, die Technik, so zu schreiben, dass sich dem Leser automatisch eine erzählte Welt, eine Diegese, präsentiert, ohne dass er zunächst mit Phänomenen der Textebene zu kämpfen hätte.“ (Baßler: Populärer Realismus, S. 91) Siehe hierzu ferner Baßler: Realismus - Serialität - Fantastik, S. 43. Auch Baßlers Verwendung des Fantastik-Begriffs ist nicht unproblematisch, da er im Grunde nur die genreförmige Fantasy-Literatur greift.

536 Baßler: Moderne und Postmoderne, S. 450.

537 Baßler: Realismus - Serialität - Fantastik, S. 44. 
hend beizupflichten haben: Ein Großteil der Werke aus dem Bereich von Fantasy oder Science-Fiction erhebt weder einen Anspruch auf konkrete realweltliche Referenzialisierbarkeit noch auf politische Relevanz (was freilich keinen prinzipiellen Einwand gegen diese Werke bedeuten muss ${ }^{538}$ ). Für andere Bereiche der amimetischen Gegenwartskunst jedoch erscheint Baßlers Verdikt weit weniger überzeugend. Während in der Zeit nach 1945 und selbst noch nach der Wende die Produktion politisch-realistischer und apolitisch-fantastischer Literatur weitgehend unvermittelt nebeneinander herlief, lassen sich in der Gegenwart zusehends Konvergenzen zwischen politischem und nicht-realistischem Schreiben beobachten: Bei der aktuell so überaus intensiv rezipierten utopischen und dystopischen Kunst ist die politische Wirkabsicht bereits qua Genre gegeben ${ }^{539}$; alternativgeschichtlich-kontrafaktische Werke, wie sie in der Gegenwartsliteratur vermehrt entstehen, verhandeln meist schwergewichtige historische Stoffe ${ }^{540}$; und apokalyptische Werke dienen in der Gegenwart nicht selten der Problematisierung ökologischer Fragen. ${ }^{541}$ Selbst in Bezug auf historisch besonders sensible oder traumatische Themen scheint das implizite Realismus-Verdikt zusehends an Bedeutung zu verlieren. Uwe Tellkamp etwa macht in seinem 2008 erschienenen Roman Der Turm über das Ende der DDR Anleihen bei der romantisch-fantastischen Erzähltradition. ${ }^{542}$ Frank Witzels

538 Dem Vorwurf des fantastischen Eskapismus kann prinzipiell auf zwei Arten begegnet werden: Entweder kann man dessen Stichhaltigkeit abstreitet, indem man darauf hinweist, dass ja auch die fantastische Kunst niemals ohne Beziehungen zur Realität auskommt. Für Stefan Berg etwa funktioniert Fantastik ,nicht primär eskapistisch, sondern, indem sie die Krisenstruktur der Moderne mit Hilfe des phantastischen Grundkonflikts verdeutlicht und verschärft, aufklärerisch, wenngleich auch mit zumeist pessimistischem Ergebnis.“ (Stefan Berg: Schlimme Zeiten, böse Räume. Zeit- und Raumstrukturen in der phantastischen Literatur des 20. Jahrhunderts. Stuttgart 1991, S. 2) Oder aber man erklärt den Eskapismus selbst für unproblematisch. Der locus classicus für diese zweite Sichtweise ist J. R. R. Tolkiens Essay On Fairy-Stories: „I have claimed that Escape is one of the main functions of fairy-stories, and since I do not disapprove of them, it is plain that I do not accept the tone of scorn or pity with which 'escape' is now so often used: a tone for which the uses of the word outside literary criticism give no warrant at all. [...] Why should a man be scorned, if, finding himself in prison, he tries to get out and go home? Or if, when he cannot do so, he thinks and talks about other topics than jailers and prison-walls? The world outside has not become less real because the prisoner cannot see it.“ (J. R. R. Tolkien: On Fairy-Stories. In: Ders.: Tree and Leaf. Smith of Wootton Major. The Homecoming of Beorhtnoth Beorhthelm's Son. London 1975, S. 11-79, hier S. 60 f.).

539 Siehe hierzu Kapitel 14. Utopie und Dystopie als Kontrafaktik.

540 Siehe hierzu Kapitel 10. Historisches Erzählen als Kontrafaktik.

541 Vgl. Eva Horn: Zukunft als Katastrophe. Frankfurt a. M. 2014, darin besonders Kapitel 3. Das Wetter von übermorgen. Imaginationsgeschichte der Klimakatastrophe, S. 110-180.

542 Vgl. Anne Fleig: Lesen im Rekord? Uwe Tellkamps Der Turm als Bildungsroman zwischen Realismus und Fantastik. In: Silke Horstkotte / Leonhard Herrmann (Hg.): Poetiken der Gegenwart. Deutschsprachige Romane nach 2000. Berlin / Boston 2013, S. 83-98. 
2015 mit dem Deutschen Buchpreis ausgezeichneter Roman Die Erfindung der Roten Armee Fraktion durch einen manisch-depressiven Teenager im Sommer 1969 bezieht neben vielen anderen Erzählformen auch fantastische Verfahren mit ein. ${ }^{543}$ Und selbst bei der literarischen Auseinandersetzunge mit dem Holocaust wird in jüngeren Werken vermehrt auf Formen fantastischen Erzählens zurückgegriffen. ${ }^{544}$

Als ein künstlerisches Verfahren, welches amimetisches und politisches Schreiben miteinander verbindet, wird nun gerade die Kontrafaktik in der Gegenwartsliteratur intensiv genutzt. (Bezeichnenderweise relativiert Moritz Baßler seine Kritik an der amimetischen Gegenwartsliteratur speziell mit Blick auf die Kontrafaktik im Werk etwa von Christian Kracht und Leif Randt. ${ }^{545}$ ) Beispiele politischer Realitätsvariationen in der Gegenwartskunst umfassen - neben den im Folgenden

543 Vgl. Karl Heinz Götze: Forever young. Über Frank Witzels Roman Die Erfindung der Roten Armee Fraktion durch einen manisch-depressiven Teenager im Sommer 1969. In: Das Argument 58/2 (2016), S. 261-266, hier S. 266.

544 Vgl. Anne-Christine Klose: „Die Zukunft ergibt sich aus der Vergangenheit.“ (Realitäts-) Effekte in aktueller zeitgeschichtlicher Jugendliteratur. In: Søren R. Fauth / Rolf Parr (Hg.): Neue Realismen in der Gegenwartsliteratur. Paderborn 2016, S. 165-180; Judith B. Kerman / John Edgar Browning (Hg.): The Fantastic in Holocaust Literature and Film. Jefferson, North Carolina 2015.

545 Baßler zufolge „zeichnet sich in Krachts Werk, ähnlich wie in den Romanen Dietmar Daths, in Pynchons Against the day oder jüngst in den Inglorious Basterds [sic] von Tarantino, die Möglichkeit eines neuen historischen Erzählens jenseits der realistischen Option ab, die Idee einer paralogisch-synthetischen Kunstwelt, die sich und uns dennoch nicht, wie die dominanten Fantasy-Welten, von den Diskursen der realen Geschichte und Gegenwart abschottet, sondern sie in etwas Neues, Artifizielles transformiert, um sie dann im Modus der Kunst zu bearbeiten.“ (Baßler: „Have a nice apocalypse!“, S. 270) Siehe auch Moritz Baßler / Heinz Drügh: Schimmernder Dunst. Konsumrealismus und die paralogischen Pop-Potenziale. In: POP. Kultur und Kritik 1 (2012), S. 60-65; Baßler: Neu-Bern, CobyCounty, Herbertshöhe. Baßlers Begriff des ,parahistorischen Erzählens“ respektive des ,Kontrafaktischen“ ist dabei ähnlich überinklusiv wie sein Begriff des ,Realismus‘ oder der ,Fantastik‘. So schreibt Baßler etwa einerseits über „[o]ffenkundig Kontrafaktisches“ (Baßler: „Have a nice apocalypse!“, S. 263) in Christian Krachts Faserland (allerdings mit fragwürdigem Textbeleg) und bezeichnet Krachts Ich werde hier sein im Sonnenschein und im Schatten als „explizit parahistorische[n] Roman“ (ebd., S. 263). Mit Blick auf Richard Kellys Soutland Tales betont Baßler dann aber, dass die „Anspielung auf George W. Bushs kontrafaktische Begründung für den Irakkrieg [...] das parahistorische Potenzial der Mediengesellschaft nur allzu deutlich [macht]“ (ebd., S. 272). Insgesamt werden damit Lüge/Postfaktizität, faktuale Kontrafaktizität und fiktionale Kontrafaktik auf problematische Weise entdifferenziert. Als Rückhalt für seine Überlegungen zum parahistorischen Erzählen bezieht sich Baßler aus der einschlägigen Forschung einzig auf die fiktionstheoretisch fragwürdigen Überlegungen von Uwe Durst (ebd., S. 261, Anm. 11). Siehe für eine Kritik an Dursts strukturalistischem Bestimmungsversuch des parahistorischen Erzählens Kapitel 4.3.5. Transfiktionale Doppelreferenz. 
noch ausführlich zu diskutierenden Werken von Christian Kracht, Kathrin Röggla, Juli Zeh und Leif Randt - Karen Duves Ökologie-Dystopie Macht (2016), Marc-Uwe Klings „Zukunftssatire“546 QualityLand (2017), Andreas Eschbachs Kombination aus Alternativgeschichte und Tech-Dystopie in NSA - Nationales Sicherheitsamt (2018) oder Elfriede Jelineks kontrafaktische Behandlung der Medienberichterstattung im Stück Winterreise (2011), ferner aus der nicht-deutschsprachigen Kunstproduktion die Serienadaption von Philip K. Dicks The Man in the High Castle (20152019), Quentin Tarantinos kontrafaktische Filme Inglourious Basterds (2009) und Once Upon a Time in Hollywood (2019), Dave Eggers' The Circle (2013) oder Michel Houellebecqs kontrovers diskutierte Vision einer Islamisierung Frankreichs im Roman Unterwerfung (2015).

Mit Blick auf die Gegenwartsliteratur scheint die Opposition zwischen politischem Realismus auf der einen und fantastischem Eskapismus auf der anderen Seite zusehends an Überzeugungskraft zu verlieren. Die (ohnehin immer prekäre) Trennung von politischer und apolitischer Kunst wird man in der Gegenwartskunst kaum plausibel entlang der Grenze von realistischem und nichtrealistischem Erzählen vornehmen können. Stattdessen wird man anerkennen müssen, dass sich in der Gegenwart auch und gerade amimetische Erzählverfahren als literarische Formen zur Verhandlung politischer Fragestellungen als produktiv erweisen, wobei den politischen Realitätsvariationen einer Kontrafaktik der Gegenwart hier besondere Bedeutung zukommt.

546 Kling: QualityLand, Klappentext, Buchdeckel vorne, Innenseite. 